\title{
Asymmetric multilevel topology for photovoltaic energy injection to microgrids
}

\author{
Javier Muñoz, Patricio Gaisse, \\ Carlos Baier, Marco Rivera \\ Department of Industrial Technologies \\ Universidad de Talca \\ Talca, CHILE \\ jamunoz@utalca.cl
}

\author{
Raúl Gregor \\ Lab. of Power and Control Systems \\ Universidad Nacional de Asunción \\ Luque, CP 2060, Paraguay \\ rgregor@ing.una.py
}

\author{
Pericle Zanchetta \\ Department of Electrical and Electronic \\ Engineering \\ University of Nottingham, U.K. \\ Pericle.Zanchetta@nottingham.ac.uk
}

\begin{abstract}
The massive penetration of renewable energy sources in the utility grid has emerged as the solution to obtain clean energy in modern electric systems, which are gradually replacing their generators that produce $\mathrm{CO}_{2}$ emissions to achieve a sustainable growing. Power electronics is quite relevant in the deep penetration of renewable energy, because the use of such equipment is mandatory to integrate these new resources with the existing facilities. In order to reach higher power ranges, multilevel topologies are the state-of-the-art solution, due to the limited rating of the actual semiconductor devices. Furthermore, latest trends show that asymmetric multilevel configurations are an attractive technology to connect directly the power converters to the grid. This paper analyze the photovoltaic energy injection to microgrids using a hybrid approach that mixes the existing topologies: string, multistring and central inverter to implement an asymmetric structure that generate highly sinusoidal resulting waveforms. This document includes a simple analysis of the proposed configuration and highlights the advantages of using an asymmetric converter, supported with stationary and dynamic simulated results.
\end{abstract}

Keywords-Asymmetric Multilevel Converters, Photovoltaic Energy Injection, Microgrids.

\section{INTRODUCTION}

According with the actual technological moment, multilevel converters are the most widespread solution to overcome the issues related to voltage and current ratings of the semiconductor devices in high and medium power applications [1][2]. In fact, commercially available converters for high and medium voltage systems are mostly based on Neutral Point Clamped (NPC), Cascaded H-Bridge (CHB) and Modular Multilevel Converter (MMC) topologies [3]-[5].

In the last years, a novel technological trend has grown in order to improve the performance of the multilevel converters, which is related with the asymmetric distribution of power among the modules [6]-[8]. This approach was firstly proposed several years ago [9] and it has slowly gained importance in recent years in different applications [10]-[13]. In the field of PV converters, asymmetric structures have not been widely studied yet. In fact, only few publications can be found related to the topic [14][16].

In this work, not only asymmetric but also hybrid structures are proposed to inject PV energy to microgrids. The main idea is to mix the existing configurations of PV converters in order to implement an asymmetric multilevel topology, as depicted in Fig. 1. The proposal considers grouping the solar panels in asymmetric arrays, suitable to use together the string, multistring and central inverter approaches. Thanks to the proper modulation of the unequal DC voltages, it is expected to obtain highly sinusoidal resulting waveforms in the converter output and therefore, the proposed structure will allow interfacing high power PV systems directly to the microgrid with a reduced filter size.

According to Fig. 1, the central inverter module can be switched at very low frequency, which implies that the commutation losses will be reduced as compared to a typical central inverter topology. In this approach the central inverter will handle most of the total power; for instance in a topology with a DC voltage ratio 9:3:1 (as shown in Fig. 1), nearly the $70 \%$ of the power is delivered by the central inverter [9]. Hence, a reduction of its commutating frequency will have an important impact in the overall efficiency of the converter.

The multistring and string converters operate with lesser power, e.g., with approximately $24 \%$ and $8 \%$ for the 9:3:1 ratio [9], shown in Fig. 1. These modules consider high switching frequencies; however, this should not have a significant impact on the overall efficiency of the converter due to the low power handled by them, as shown in some losses analyses made over asymmetric structures [17][18].

For the string and multistring stages, the use of DC/DC converters is an integral part of the structure in order to track the Maximum Power Point (MPP) of the solar panels. However, in the central inverter this stage is avoided because extra semiconductor devices imply higher switching losses due to the high power handled for this module. Therefore, the MPP in the central inverter is directly tracked by the DC/AC converter.

Therefore, the proposed approach can achieve a highly sinusoidal output waveform with reduced commutating frequencies in the modules that manage more power. The inclusion of $\mathrm{DC} / \mathrm{DC}$ converters is only considered in the low power stages in order to produce a reduced impact on the overall efficiency.

The focus of this paper lies on the control and performance of the central inverter in the hybrid topology. Particularly, the dynamic model is obtained and the proposed control strategy is tested under severe irradiance changes. 

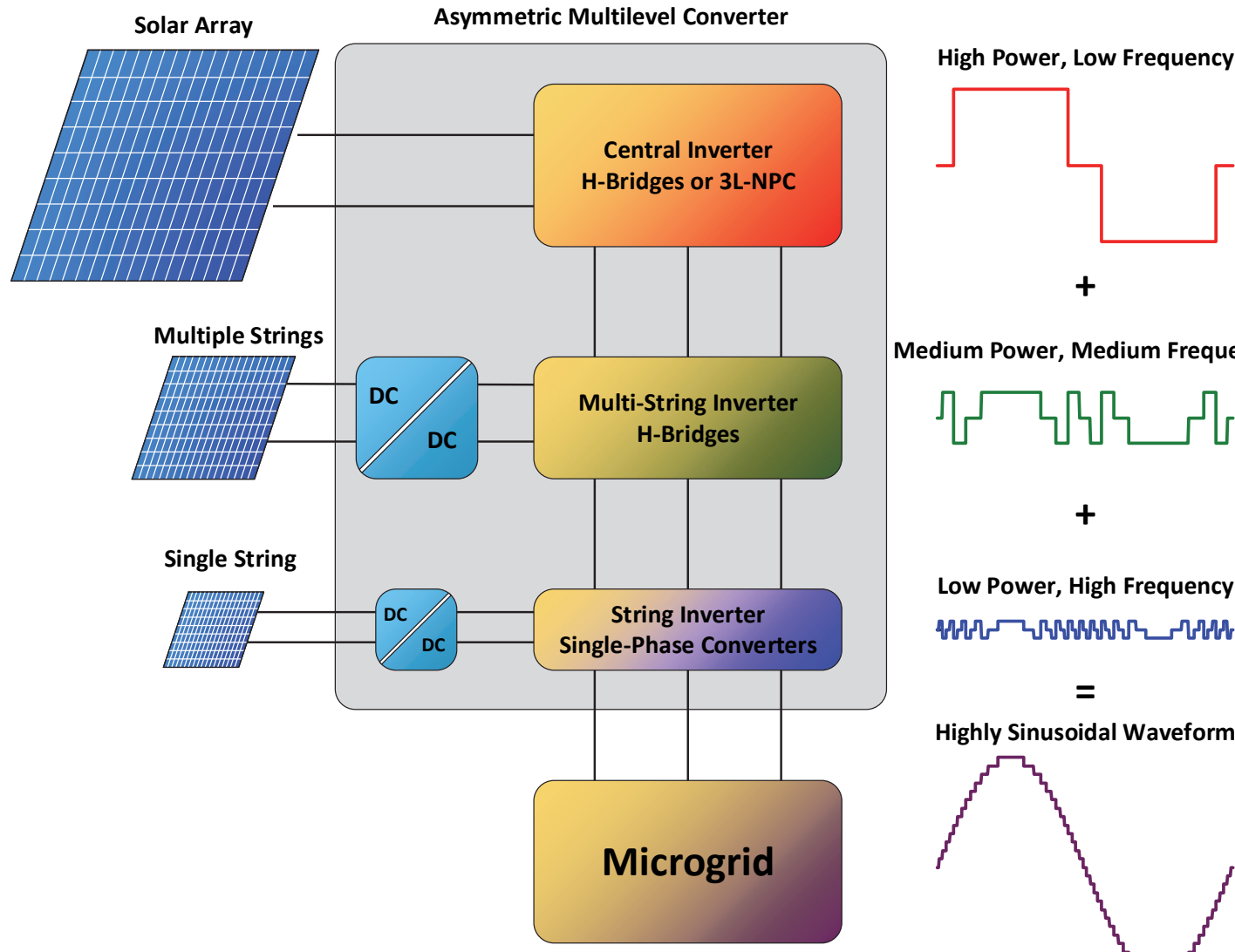

Medium Power, Medium Frequency

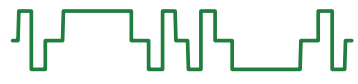

Low Power, High Frequency

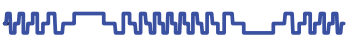

$=$

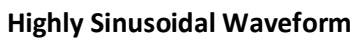

Fig. 1 Asymmetric Multilevel Converter for PV Power Injection into Microgrids.

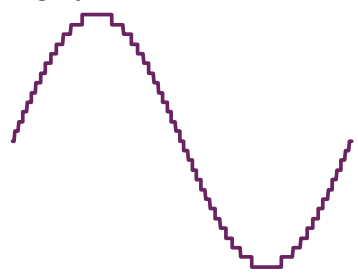

\section{THE PROPOSED APPROACH}

The schematic of the proposed topology is shown in Fig. 2, where it is possible to observe the cascade connection of three different H-bridge inverters, for each phase. Despite the series connection of the modules in the $\mathrm{AC}$ side, each power cell will handle different power levels due to the asymmetric distribution of voltages. Then, different configurations and sizes of PV panels are considered in this topology. For instance, as detailed in Fig. 2, the High Power Cell (HPC) considers a PV array, the Medium Power Cell (MPC) acts as interface for a multi-string converter and the Low Power Cell (LPC) uses a single PV string.

It is important to remark that the focus of this paper will lie on the performance of the HPC; however, there are other important issues to address that will be analyzed in future works, such as the operating region, optimized gating patterns, MPPT strategies under partial shading, just to name a few.

\section{A. Dynamic Analysis}

One of the main steps in order to achieve a successful control strategy for the HPC is to obtain the dynamic model of the system. It is possible to observe from Fig. 2 that the total AC voltage $v_{c}$ injected by the Asymmetric Multilevel Converter (AMC) can be obtained as the sum of the individual voltages in each power cell, due to the cascaded connection of the modules.

The total AC voltage injected by the converter is obtained as,

$$
v_{c}=v_{9}^{a c}+v_{3}^{a c}+v_{1}^{a c} .
$$

For modeling purposes and for the sake of simplicity, this total voltage will be expressed as,

$$
v_{c}=m_{c} V_{D C},
$$

where $m_{c}$ is the modulating signal of the converter and $V_{D C}$ is a virtual voltage obtained as the sum of the individual DC links. This is,

$$
V_{D C}=V_{9}+V_{3}+V_{1} .
$$

Therefore, to obtain the dynamic model in the AC side, a known $V_{D C}$ voltage is assumed for the converter, which value is set by the Maximum Power Point Tracking (MPPT) algorithm. Taking into account that the proposed topology use single-phase converters, the circuit diagram shown in Fig. 2 allows to state the system equation for each phase as,

$$
v_{s}=L_{c} \frac{d}{d t} i_{c}+R_{c} i_{c}+m_{c} V_{D C} .
$$




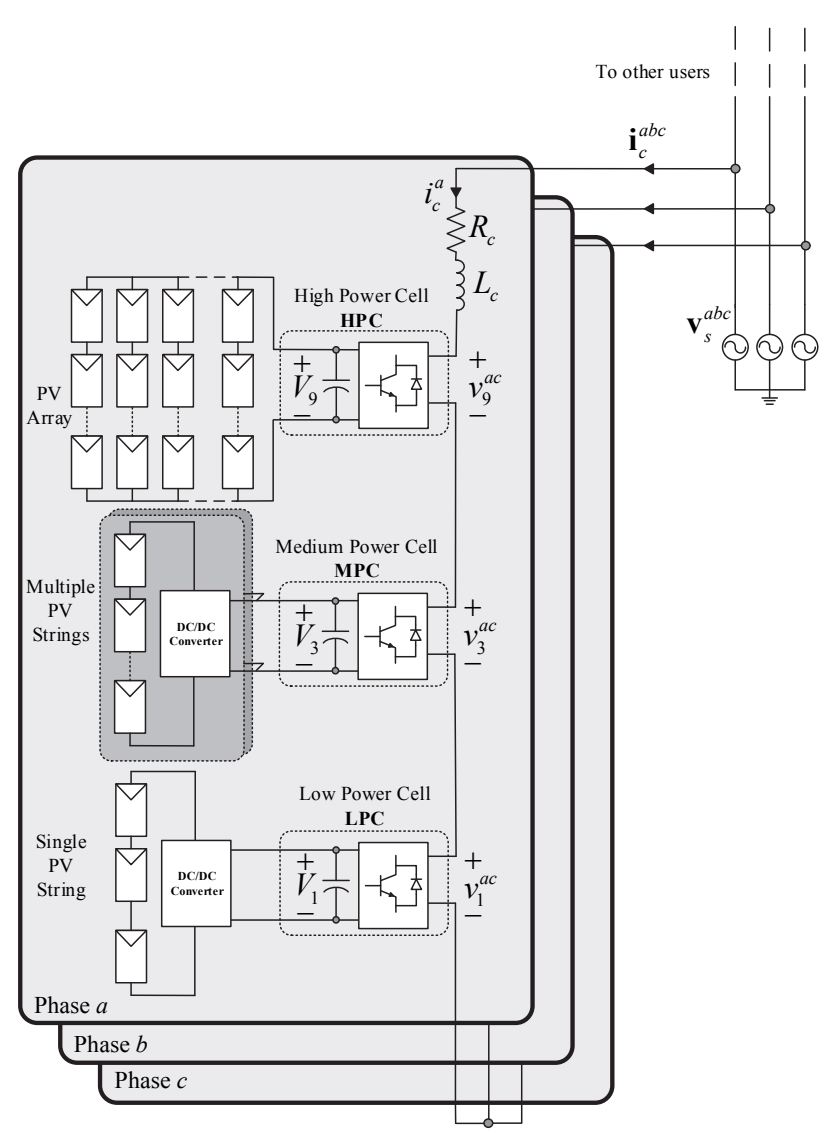

Fig. 2 Asymmetric Topology

Although the topology considers a complete three-phase system, a single-phase Park Transform is proposed, due to the single-phase nature of the cascaded converter. Therefore, the modeling equation must be $\pm 120^{\circ}$ shifted to obtain the dynamic model of the other two phases. Then, it is possible to rewrite equation (4) in the synchronous reference frame as,

$$
\begin{aligned}
& \frac{d}{d t} i_{s}^{d}=-\frac{R_{c} i_{c}^{d}}{L_{c}}+\omega_{s} i_{c}^{q}-\frac{m_{c}^{d} V_{D C}}{L_{c}}+\frac{v_{s}^{d}}{L_{c}} \\
& \frac{d}{d t} i_{s}^{q}=-\omega_{s} i_{c}^{d}-\frac{R_{c} i_{s}^{q}}{L_{c}}-\frac{m_{c}^{q} V_{D C}}{L_{c}}+\frac{v_{s}^{q}}{L_{c}}
\end{aligned}
$$

It is worth mentioning that the virtual $V_{D C}$ voltage is assumed constant because an external master control loop will be in charge to maintain it in its reference value given by the

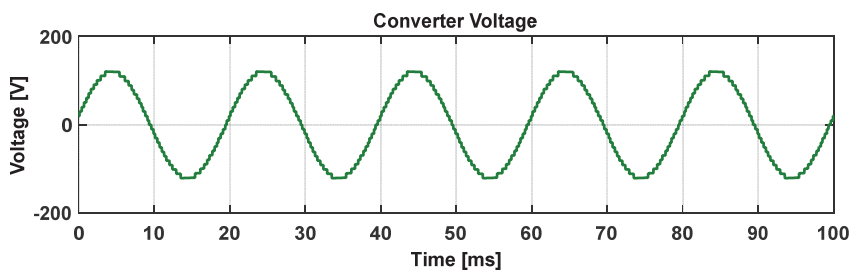

(a)
MPPT algorithm. Then, the internal current loops are responsible to generate the modulating signals in the $d q$ frame, as will be explained in the forthcoming section.

\section{B. Operating Point}

In order to stablish the operating point of the $\mathrm{AMC}$, it is necessary to calculate the modulating signals required to inject the desired active and reactive current. These values are limited due to the bounded modulating signals that should not reach the over-modulation region in order to achieve the expected low harmonic distortion, characteristic of an asymmetric structure. The steady state value for the modulating signal for a given operating point can be found solving,

$$
\dot{\mathbf{x}}=\mathbf{f}(\mathbf{x}, \mathbf{u})
$$

such that,

$$
\mathbf{0}=\mathbf{f}\left(\mathbf{x}_{\mathbf{0}}, \mathbf{u}_{\mathbf{0}}\right),
$$

where $\mathbf{x}$ is the state vector that in this case are the converter currents and $\mathbf{u}$ is the converter input, which corresponds to the modulating signals in the $d q$ frame. The subscript $\mathbf{o}$ is used to indicate an arbitrary operating condition. The boundary is set by the maximum modulating signals in order to avoid overmodulation. The general expression of (7) is solved for the model given by (5), leading to (8).

$$
\begin{aligned}
& M_{c}^{d}=\frac{-R_{c} I_{c}^{d}+\omega_{s} L_{c} I_{c}^{q}+V_{s}^{d}}{V_{D C}} \\
& M_{c}^{q}=\frac{-\omega_{s} L_{c} I_{c}^{d}-R_{c} I_{c}^{q}+V_{s}^{q}}{V_{D C}} .
\end{aligned}
$$

Therefore, in order to analyze whether an operating condition can be reached, for an imposed converter current, the following expression must be fulfilled,

$$
\left(M_{c}^{d}\right)^{2}+\left(M_{c}^{q}\right)^{2} \leq 1
$$

An open loop simulation was performed, with the aim of evaluating the obtained model. The system parameters are depicted in Table I and the key waveforms are shown in Fig. 3 . It is possible to observe that the voltage and current in the converter are in phase, as the $q$ component of the converter current was set to zero. These waveforms confirm the properness of the model, which is the basis to propose a suitable control scheme.

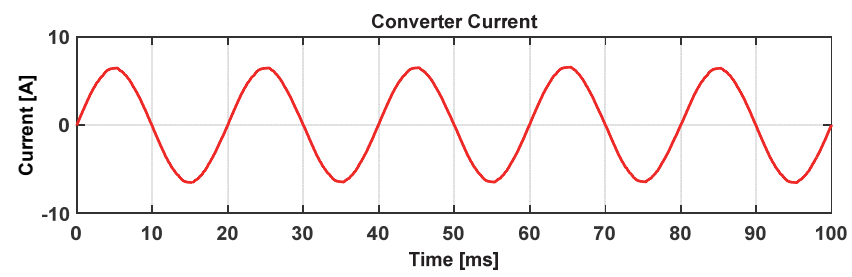

(b)

Fig. 3 Steady state waveforms of the Asymmetric Multilevel Converter (a) Total Generated AC Voltage, (b) Total Injected AC Current. 
TABLE I PARAMETERS

\begin{tabular}{ccrc}
\hline \hline Parameter & Description & \multicolumn{2}{c}{ Value } \\
\hline$R_{C}$ & Resistor of the converter passive filter & 0.2 & $\Omega$ \\
$L_{C}$ & Inductor of the converter passive filter & 10 & $\mathrm{mH}$ \\
$f_{s}$ & Microgrid System Frequency & 50 & $\mathrm{~Hz}$ \\
$V_{s}$ & Microgrid Nominal Voltage & 110 & $\mathrm{~V}_{\mathrm{RMS}}$ \\
\hline \hline
\end{tabular}

\section{Modulation Strategy AND CONTROL SCHEME}

In order to obtain the desired amplitude and phase of the injected current it is necessary to develop a proper modulation strategy that allows the generation of any arbitrary sinusoidal shape (within the physical restrictions of the converter). In this work, the modulation strategy is based on a simple look-up table, where all the valid states of the converter are stored.

Table II shows the 27 possible combination of voltages of the converter; where HPC, MPC and LPC, stand for the commutation states of the High Power Cell, Medium Power Cell and Low Power Cell, respectively. In order to generate a reference voltage, the modulation index is amplified by 13 (considering that the modulating signal goes from -1 to +1 ), and then quantized in the 27 levels depicted in Table II. The resulting value is used as the input of the look-up table, from where the state of each converter is obtained. Furthermore, to distribute equally the commutation losses, the redundancy of the zero states is shared between the negative and positive levels of the waveforms, while the zero level is alternated.

Further analyses of this modulating technique are developed in [19] and more advanced strategies can be found in the technical literature as well [20], but this work considers a simple commutation algorithm - as the described above - in order to focus the analysis on the performance of the proposed topology.

The control scheme of the HPC include a master-slave loop, where the internal controllers are meant to regulate the $d q$ components of the converter current, while the external loop regulates the DC voltage, as shown in Fig. 4. For the MPC and the LPC, the control is made with dedicated DC/DC converters, which are required due to the fixed patterns of the H-Bridges, necessary to achieve the characteristic 27-level waveform. Then, MPC and LPC are considered stiff sources in
TABLE II LOOK-UP TABLE

\begin{tabular}{cccccc}
\hline \hline State & Level & HPC & MPC & LPC & Converter Voltage \\
\hline 1 & -13 & -1 & -1 & -1 & $-\mathrm{V}_{9}-\mathrm{V}_{3}-\mathrm{V}_{1}$ \\
2 & -12 & -1 & -1 & 0 & $-\mathrm{V}_{9}-\mathrm{V}_{3}$ \\
3 & -11 & -1 & -1 & +1 & $-\mathrm{V}_{9}-\mathrm{V}_{3}+\mathrm{V}_{1}$ \\
4 & -10 & -1 & 0 & -1 & $-\mathrm{V}_{9}-\mathrm{V}_{1}$ \\
5 & -9 & -1 & 0 & 0 & $-\mathrm{V}_{9}$ \\
6 & -8 & -1 & 0 & +1 & $-\mathrm{V}_{9}+\mathrm{V}_{1}$ \\
7 & -7 & -1 & +1 & -1 & $-\mathrm{V}_{9}+\mathrm{V}_{3}-\mathrm{V}_{1}$ \\
8 & -6 & -1 & +1 & 0 & $-\mathrm{V}_{9}+\mathrm{V}_{3}$ \\
9 & -5 & -1 & +1 & +1 & $-\mathrm{V}_{9}+\mathrm{V}_{3}+\mathrm{V}_{1}$ \\
10 & -4 & 0 & -1 & -1 & $-\mathrm{V}_{3}-\mathrm{V}_{1}$ \\
11 & -3 & 0 & -1 & 0 & $-\mathrm{V}_{3}$ \\
12 & -2 & 0 & -1 & +1 & $-\mathrm{V}_{3}+\mathrm{V}_{1}$ \\
13 & -1 & 0 & 0 & -1 & $-\mathrm{V}_{1}$ \\
14 & 0 & 0 & 0 & 0 & 0 \\
15 & 1 & 0 & 0 & +1 & $\mathrm{~V}_{1}$ \\
16 & 2 & 0 & +1 & -1 & $+\mathrm{V}_{3}-\mathrm{V}_{1}$ \\
17 & 3 & 0 & +1 & 0 & $+\mathrm{V}_{3}$ \\
18 & 4 & 0 & +1 & +1 & $+\mathrm{V}_{3}+\mathrm{V}_{1}$ \\
19 & 5 & +1 & -1 & -1 & $+\mathrm{V}_{9}-\mathrm{V}_{3}-\mathrm{V}_{1}$ \\
20 & 6 & +1 & -1 & 0 & $+\mathrm{V}_{9}-\mathrm{V}_{3}$ \\
21 & 7 & +1 & -1 & +1 & $+\mathrm{V}_{9}-\mathrm{V}_{3}+\mathrm{V}_{1}$ \\
22 & 8 & +1 & 0 & -1 & $+\mathrm{V}_{9}-\mathrm{V}_{1}$ \\
23 & 9 & +1 & 0 & 0 & $+\mathrm{V}_{9}$ \\
24 & 10 & +1 & 0 & +1 & $+\mathrm{V}_{9}+\mathrm{V}_{1}$ \\
25 & 11 & +1 & +1 & -1 & $+\mathrm{V}_{9}+\mathrm{V}_{3}-\mathrm{V}_{1}$ \\
26 & 12 & +1 & +1 & 0 & $+\mathrm{V}_{9}+\mathrm{V}_{3}$ \\
27 & 13 & +1 & +1 & +1 & $+\mathrm{V}_{9}+\mathrm{V}_{3}+\mathrm{V}_{1}$ \\
\hline \hline
\end{tabular}

this analysis. Therefore, as highlighted earlier, the analysis will be focused on the control algorithm of the High Power Cells.

Fig. 4 shows that the reference of the direct current is generated with the DC voltage control block that considers a PI controller. As the Park transformation is synchronized with the source voltage, the direct current is associated with the active power generated by the PV array. In the case of the quadrature current, it is set to zero; however a different value can be imposed according to the reactive power requirements of the microgrid, as well as the available capacity of the converter.

On the other hand, the current controllers in Fig. 4 generate the direct and quadrature components of the signals used in the modulation stage. This control block was implemented with a linear control strategy. In fact, PI

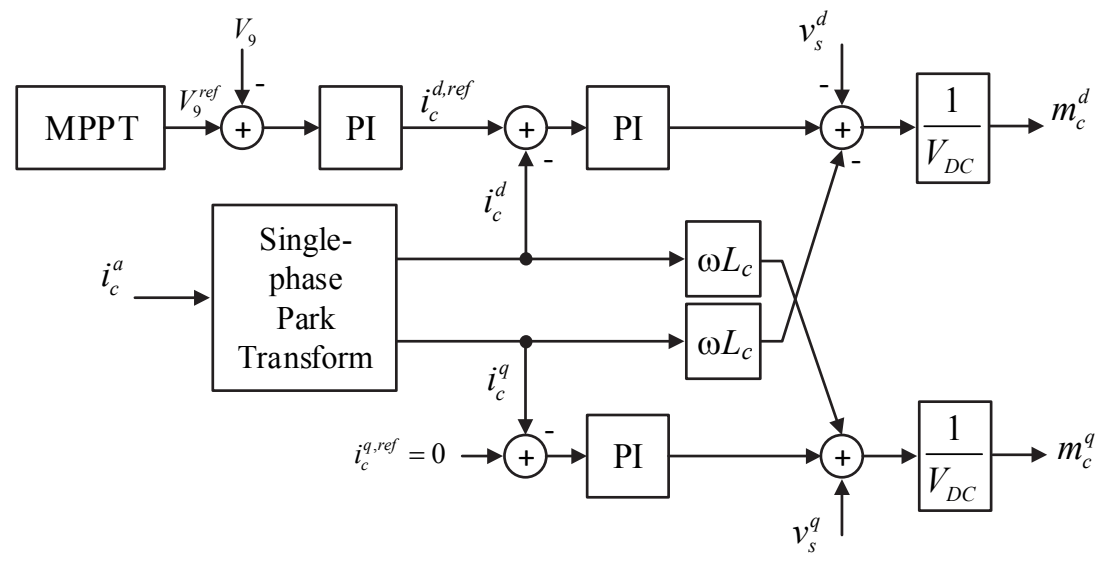

Fig. 4 Control strategy for each phase 
controllers are quite suitable in this scheme, because $d q$ components of the converter currents are DC quantities and therefore simple PI controllers can achieve zero steady state error with an acceptable dynamic performance. Hence, Fig. 4 shows the typical linear current controller in the synchronous reference frame that is used to control direct and quadrature currents independently, obtaining satisfactory dynamic response, as will be demonstrated in the next section. In Fig. 4 the coupled terms of the dynamic model developed in (5), are canceled out in the control strategy, which allows proposing single-variable PI controllers for each loop. A feedforward term coming from the source voltage $v_{s}$ is added to the controllers output to compensate disturbances in the grid.

\section{Simulated Results}

Using the proposed modulating and control techniques, it is expected to obtain highly sinusoidal waveforms in the converter output. Furthermore, the controlled injection of active and reactive current will ensure the MPPT of the PV modules, as well as the eventual reactive power compensation requested by the microgrid.

A closed loop simulation was performed in the PSIM software and the main results are summarized in Fig. 5 to Fig. 7. According to the waveforms of Fig. 5, it is possible to observe that the HPC is capable to maintain the DC voltage in its reference of $90 \mathrm{~V}$, regardless the step-down disturbance from $1000 \mathrm{~W} / \mathrm{m}^{2}$ to $500 \mathrm{~W} / \mathrm{m}^{2}$ in the solar irradiance of the PV modules of all phases, at $t=200 \mathrm{~ms}$. It is important to remark, that the partial shading effect is not included in the analyses; then, all phases are balanced.

Fig. 6 (a) shows the dynamic behavior of the direct components of the converter current, for the three phases for the same irradiance change at $200 \mathrm{~ms}$. As described in the control scheme in Fig. 4, these currents define the value of the modulating signals for each phase, which are shown in the plot of Fig. 6 (b). In this case, the reactive currents are set to zero; then, the converter only injects to the microgrid the active power coming from the PV modules.

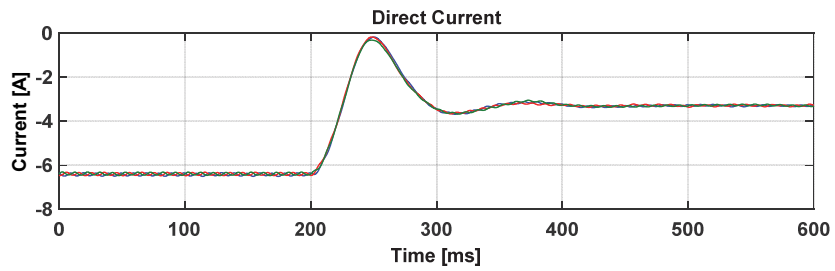

(a)

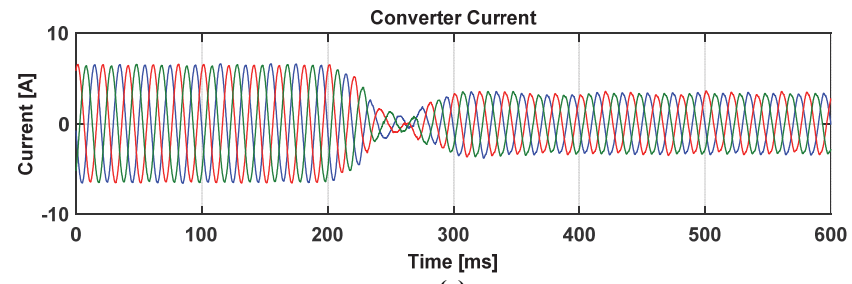

(c)

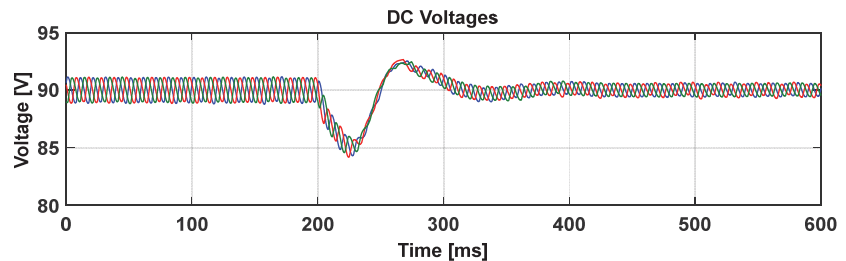

(a)

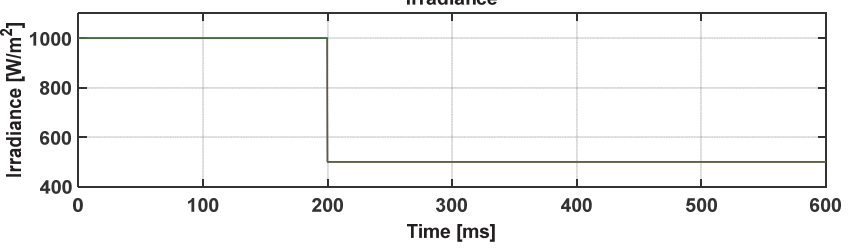

(b)

Fig. 5 Key DC variables of the Asymmetric Multilevel Converter (a) DC Voltages of the High Power Cells (b) Irradiance of the PV modules.

On the other hand, the reference for the direct component of the converter current is given by the master DC voltage control. As shown in Fig. 6 (a), these currents are negatives, which can be easily explained considering the sign convention defined in Fig. 2 that establish energy consumption for positive current and generation for negative values. As expected, the magnitude of the direct current decreases due to lower irradiance in the PV modules.

Fig. 6 (c)-(d) illustrate the transient of the converter currents and voltages when the irradiance change appears. Consequently with the results of Fig. 6 (a), the amplitude of the injected current decreases due to the less amount of power produced by the PV modules. It can be seen that the converter reach its new operating condition in a couple of cycles, where the THD of the steady state voltage is $3.4 \%$ and for the current is $0.9978 \%$ thanks to the inductive filter.

The start-up process is also shown in order to analyze the asymmetric distribution of the DC voltages. The key waveforms are illustrated in Fig. 7, where it can be observed that the DC voltages reach the desired 9:3:1 ratio after a transient period due to the start-up of the control algorithms.

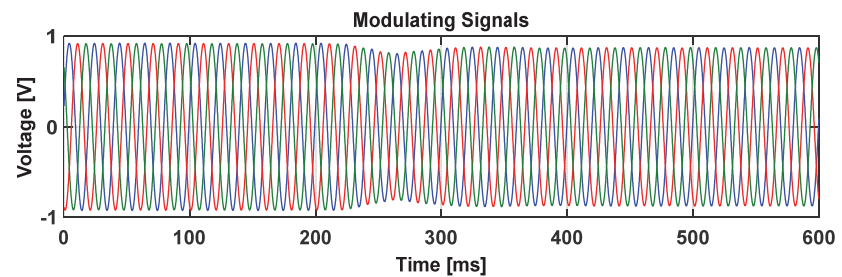

(b)

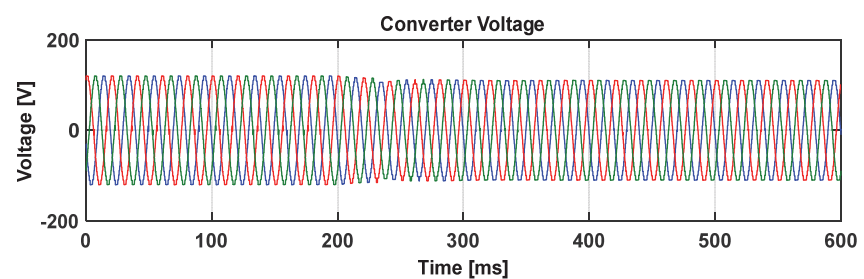

(d)

Fig. 6 Key AC waveforms of the Asymmetric Multilevel Converter (a) Direct Currents, (b) Modulating Signals, (c) Currents, (d) Voltages. 


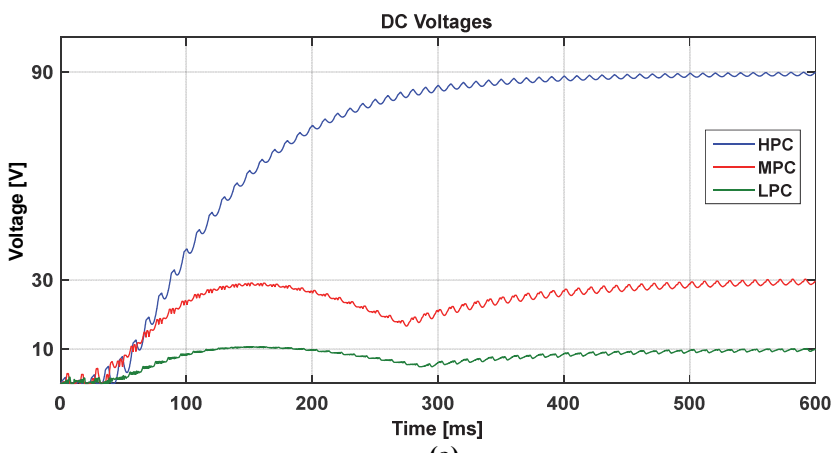

(a)

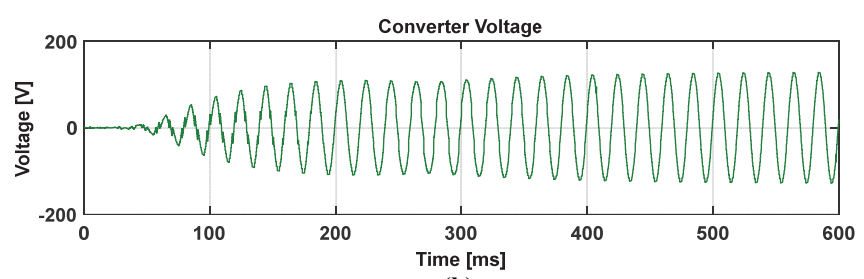

(b)

Fig. 7 Dynamic Waveforms during Start-Up

(a) DC Voltages for each stage (b) AC Injected Voltage.

Around the $300 \mathrm{~ms}$, there is a notorious change in the DC voltages of the low power modules (MPC and LPC), which is due to the activation of the DC/DC converters of these stages. Fig. 7 (b) shows the injected AC voltage that is highly sinusoidal with 27 levels, once the DC ratio is stabilized.

As explained earlier, the HPC uses a master control scheme in order to track the MPP. The remaining stages use a DC/DC converter to obtain the maximum power from the solar strings and to ensure the requested voltage ratio. It is important to remark that tracking the MPP is suitable when the microgrid is connected to the utility grid, which is in charge to balance the power flow between sources and loads. For the islanding operating mode, the control strategy should be modified, aiming to collaborate with the microgrid stability.

\section{CONCLUSIONS}

This paper presents a novel approach that mixes the central inverter, the multistring and the string topologies in order to generate a highly sinusoidal AC waveform, characteristic of asymmetric multilevel converters. This work includes a detailed explanation of the converter operation, a simple analysis of the control strategy and the modulating technique, and a comprehensive set of stationary and dynamic results to illustrate that this approach can be an attractive alternative to classical symmetric multilevel converters for PV integration to microgrids. This work shows that the inclusion of asymmetric multilevel structures to integrate PV energy to microgrids is feasible. Thanks to this approach, it is possible to use the advantages of low power modules (string and multistring converters) to overcome the main drawbacks of high power modules (central inverter approach). Using proper control algorithms and modulating schemes is possible to simultaneously track the MPP and maintain the required DC voltage ratio in order to achieve highly sinusoidal waveforms.

\section{REFERENCES}

[1] Kouro, S.; Malinowski, M.; Gopakumar, K.; Pou, J.; Franquelo, L.G.; Bin Wu; Rodriguez, J.; Perez, M.A.; Leon, J.I., "Recent Advances and Industrial Applications of Multilevel Converters," IEEE Trans. on Ind. Electron., vol.57, no.8, pp.2553,2580, Aug. 2010.

[2] Sergio Alberto Gonzalez, Santiago Andres Verne, Maria Ines Valla, Multilevel Converters for Industrial Applications, CRC Press, 2013.

[3] ABB. [Online]. Available: www.abb.com

[4] SIEMENS. [Online]. Available: www.siemens.com

[5] Schneider-Electric. [Online]. Available: www.schneider-electric.com

[6] Pereda, J.; Dixon, J., "Cascaded Multilevel Converters: Optimal Asymmetries and Floating Capacitor Control," Industrial Electronics, IEEE Transactions on, vol.60, no.11, pp.4784,4793, Nov. 2013.

[7] S. M. Barakati, A. Khoshkbar Sadigh and E. Mokhtarpour, "Voltage sag and swell compensation with DVR based on asymmetrical cascade multicell converter," North American Power Symposium (NAPS), 2011, Boston, MA, 2011, pp. 1-7.

[8] Perez, M.A.; Cortes, P.; Rodriguez, J., "Predictive Control Algorithm Technique for Multilevel Asymmetric Cascaded H-Bridge Inverters," Industrial Electronics, IEEE Transactions on, vol.55, no.12, pp.4354, 4361, Dec. 2008.

[9] Dixon, J.; Moran, L., "Multilevel inverter, based on multi-stage connection of three-level converters scaled in power of three," IECON 02 [Industrial Electronics Society, IEEE 2002 28th Annual Conference of the], vol.2, no., pp.886,891 vol.2, 5-8 Nov. 2002.

[10] Boobalan, S.; Dhanasekaran, R., "Hybrid topology of asymmetric cascaded multilevel inverter with renewable energy sources," Advanced Communication Control and Computing Technologies (ICACCCT), 2014 International Conference on , vol., no., pp.1046,1051, 8-10 May 2014.

[11] Elias, M.F.M.; Abd Rahim, N.; Hew Wooi Ping; Uddin, M.N., "Asymmetrical Cascaded Multilevel Inverter Based on TransistorClamped H-Bridge Power Cell," Industry Applications, IEEE Transactions on , vol.50, no.6, pp.4281,4288, Nov.-Dec. 2014

[12] Veenstra, M.; Rufer, A., "Control of a hybrid asymmetric multilevel inverter for competitive medium-voltage industrial drives," Industry Applications, IEEE Transactions on , vol.41, no.2, pp.655,664, MarchApril 2005

[13] Flores, P.; Dixon, J.; Ortuzar, M.; Carmi, R.; Barriuso, P.; Moran, L., "Static Var Compensator and Active Power Filter With Power Injection Capability, Using 27-Level Inverters and Photovoltaic Cells," IEEE Trans. on Ind. Electron., vol.56, no.1, pp.130,138, Jan. 2009.

[14] Draxe, K.P.; Ranjana, M.S.B.; Pandav, K.M., "A cascaded asymmetric multilevel inverter with minimum number of switches for solar applications," Power and Energy Systems Conference: Towards Sustainable Energy, 2014, vol., no., pp.1,6, 13-15 March 2014.

[15] M. A. Perez and S. Kouro, "Asymmetric cascaded converter for solar PV applications," 2014 IEEE 23rd International Symposium on Industrial Electronics (ISIE), Istanbul, 2014, pp. 2484-2489.

[16] Malathy, S.; Ramaprabha, R.; Mathur, B.L., "Asymmetrical multilevel inverters for partially shaded PV systems," Circuits, Power and Computing Technologies (ICCPCT), 2013 International Conference on, vol., no., pp.579,583, 20-21 March 2013.

[17] Kouro, S.; Bernal, R.; Miranda, H.; Rodriguez, J.; Pontt, J., "Direct Torque Control With Reduced Switching Losses for Asymmetric Multilevel Inverter Fed Induction Motor Drives," Industry Applications Conference, 2006. 41st IAS Annual Meeting. Conference Record of the 2006 IEEE, vol.5, no., pp.2441,2446, 8-12 Oct. 2006.

[18] Munoz, J.; Baier, C.; Espinoza, J.; Rivera, M.; Guzman, J.; Rohten, J., "Switching losses analysis of an asymmetric multilevel Shunt Active Power Filter," Industrial Electronics Society, IECON 2013 - 39th Annual Conference of the IEEE, vol., no., pp.8534,8539, 10-13 Nov. 2013.

[19] M. Perez, J. Rodriguez, J. Pontt and S. Kouro, "Power Distribution in Hybrid Multi-cell Converter with Nearest Level Modulation," 2007 IEEE International Symposium on Industrial Electronics, Vigo, 2007, pp. 736-741.

[20] M. Rotella, G. Penailillo, J. Pereda and J. Dixon, "PWM Method to Eliminate Power Sources in a Nonredundant 27-Level Inverter for Machine Drive Applications," in IEEE Transactions on Industrial Electronics, vol. 56, no. 1, pp. 194-201, Jan. 2009. 